\title{
A complex cell
}

\section{New data redefine macrophages as diverse, polyfunctional and plastic cells that respond to the needs of the tissue at steady state and during disturbed homeostasis.}

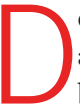
espite a historical wealth of knowledge about macrophages, recent advances have revealed new and surprising aspects about their biology and function. Long known for their phagocytic activity and their role in immune responses, macrophages are now recognized as serving important functions in systemic metabolism, cold adaptation, and tissue homeostasis and development. A key feature of macrophages is their ability to adapt to the stimuli in their environment. Macrophages not only respond with diverse phenotypes but also can switch from one functional phenotype to another, in a dynamic and continuous adaptation to the needs of the tissue. In this issue of Nature Immunology, we provide a series of specially commissioned articles to review the most recent progress in understanding of the ontogeny, diversity and activation plasticity of macrophages and the regulatory programs that control these aspects of macrophage biology.

Contrary to historical views that tissue macrophages are maintained by a constant supply from the bone marrow through circulating blood monocyte intermediates, it is now known that two distinct populations of macrophages, distinguished by their progenitors, developmental history and mechanisms of turnover and maintenance, coexist in adult tissue. Geissmann and Gomez Perdiguero discuss this layered system, in which 'resident' macrophages that develop in the embryo and persist in adult tissue independently of bone marrow hematopoiesis coexist with monocyte-derived macrophages that originate and renew from the adult bone marrow. The authors review early and recent work leading to the identification of three successive but overlapping waves of progenitor cells that can give rise to fetal macrophages, and they detail how these can be distinguished by anatomical site of emergence, differentiation potential and transcription factor dependence.

Medzhitov outlines two general principles that can help to conceptualize the view of macrophages as essential components of tissues in metazoans. First, the general tendency for progressive 'division of labor' indicates that as cells diversify during evolution, they perform fewer and more specialized functions and subsequently delegate secondary functions to accessory cells. Tissue macrophages are such accessory cells that perform generic functions, such as the removal of apoptotic cells; highly specialized functions, such as bone resorption; or functions on demand, such as defense and repair. As the author points out, macrophages can act as accessory cells even to tumors. Second, the phenotype of tissue-resident macrophages is determined by the integration of tissue-identity signals and functional demands and thus is a combination of differentiation and polarization programs. A particular program can be fixed in some tissue macrophages and induced in others, depending on the needs and characteristics of the tissue. The overall phenotype is thus established by hierarchies of lineage, tissue identity and on-demand transcription modules.

Amit, Winter and Jung discuss the variety of environmental conditions that shape and maintain macrophage identity, as well as the mechanisms that mediate such 'imprinting'. Macrophages acquire attributes tailored specifically to the homeostatic needs of the tissue in which they reside, such as the provision of niche-specific factors or morphological or functional specialization. The specific conditions in each tissue, such as an abundance of heme in the spleen or of the soluble factor TGF- $\beta$ in the brain, are critical regulators of these tissue signatures. The authors argue that macrophage identity in the tissues is determined by a combination of lineage- and tissue-specific transcription factors and that the macrophage regulatory network has evolved to accommodate plasticity.

Glass and Natoli discuss the present understanding of the mechanisms underlying priming and signal-dependent activation of macrophages and broadly review basic chromatin and epigenetic features that explain the effect of lineage-specific, pre-established genomic landscapes on determining specific activation responses or tissue-specific profiles. The authors point out that coupling individual signals to specific outputs depends on the combination of selective activation of a limited number of signaling pathways and transcription factors and the repertoire of accessible genomic sites preexistent in that particular cell. This explains how specific signals direct distinct patterns of expression required for the specialized function of different cell types.

In a Perspective, Ginhoux and colleagues discuss how such recent advances in macrophage development, activation and functional diversity shape the understanding of macrophage biology and drive new models of activation and classification, away from the classical dichotomous categorization as M1 and M2 macrophages and into more complex models that can accommodate the continuum of transcriptional and functional states observed in vivo. The authors also discuss how high-resolution analysis of macrophage origin and activation, such as fate mapping, single-cell RNA sequencing, epigenetic programing and mass cytometry, will help elucidate the dynamic interaction between macrophages and the tissue in homeostatic and pathological situations in vivo.

Through their ontogeny, phenotype and function, macrophages are an integral part of tissues. They are present in all tissues, they have a long lifespan and self-renew. They perform housekeeping, metabolic and tissue remodeling functions but also acquire specifically tailored attributes, such as the provision of niche-specific factors or morphological and functional specialization. They are immunosuppressive at steady state and orchestrate immune responses when homeostasis is disturbed. Given such profound connection with the tissue, it is not surprising that various pathologies, ranging from obesity to autoimmunity and neurodegenerative disease, have been linked to the dysregulation of macrophage function. Further study of the functional heterogeneity of these cells and the programs that establish and maintain their identity in the tissue will be essential for basic understanding of their biology and future clinical applications. 